Depressive und kognitive Störungen

\title{
Liegt es an der Schilddrüse?
}

- Neuropsychiatrische Symptome einer Hypothyreose sind unter L-Thyroxin-Substitution reversibel. Das ist seit Längerem belegt. Ebenso, dass auch das adulte Gehirn essenziell Schilddrüsenhormone benötigt. Ein Eingriff in den Schilddrüsenstoffwechsel beeinflusst die Stimmungslage massiv. Zwischen der TSH-Serumkonzentration und der Depressionsschwere besteht eine enge Korrelation, sagte Prof. Michael Bauer, Dresden. Hypothyreotische Patienten weisen mit 40-70\% im Vergleich zu hyperthyreotischen Patienten wesentlich häufiger depressive und kognitive Störungen und Syndrome auf. Sollte unter L-Thyroxin keine ausreichende Verbesserung eintreten, empfiehlt er die zusätzliche Gabe eines Antidepressivums. Bleiben die Störungen über einen längeren Zeitraum unbehandelt, kann es zu irreversiblen neuronalen Schädigungen kommen, warnte der Psychiater.

\section{Knoten ab $\mathbf{2}$ cm tastbar}

Bei der Diagnostik von Schilddrüsenknoten kann die Sonografie mit hochauflösenden Schallsonden bereits fokale Veränderungen von 1-2 $\mathrm{mm}$ erkennbar machen, so Dr. Jörg-Carsten Kämmer, Berlin. Bei der Differenzierung zwischen malignen und benignen Knoten sind sonografische Methoden - trotz Charakteristika wie echoarm, schlecht abgrenzbar, Mikroverkalkungen, ohne echoarme Halo nicht ausreichend. Bei begründetem Verdacht auf ein Schilddrüsenkarzinom ist eine Feinnadelpunktion notwendig. Vorgeschaltet werden sollte eine Szintigrafie, so Prof. Markus Luster, Ulm, damit gezielt nur ,kalte“ Knoten punktiert werden.

Für den Nuklearmediziner steht die Palpation bei der Schilddrüsendiagnostik nach wie vor an erster Stelle. „Alles was über zwei bis drei Zentimeter groß ist, ist gut zu tasten“, sagte Luster. Bei einem

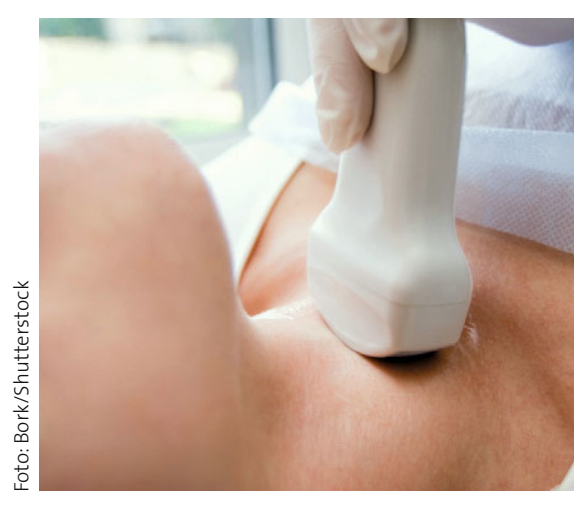

Per Ultraschall sind fokale

Veränderungen $a b$ 1-2 $\mathrm{mm}$ erkennbar.

Durchmesser von $10-20 \mathrm{~mm}$ sind $50 \%$ der Knoten tastbar. Hier hat die Sonografie einen klaren Vorteil.

- Ulrike Tietze

Quelle: Schilddrüsengespräch, Berlin, 12. Dezember 2009 (Veranstalter: Merck Pharma GmbH)

\section{Mehr Informationen}

... zu Schilddrüsenerkrankungen exklusiv für Fachkreise:

www.schilddrueseninstitut.de

\section{Strenge Blutzuckersenkung zahlt sich langfristig aus \\ Bei oraler Diabetestherapie früh auf Kombinationen setzen}

- Bei Typ-2-Diabetikern lässt sich durch eine frühe, strenge Blutzuckerkontrolle das Risiko für mikrovaskuläre Erkrankungen und Herzinfarkte sowie die Sterblichkeit signifikant reduzieren. Dr. Jens Kröger, Hamburg, belegte diese Aussage mit den Ergebnissen der zehnjährigen Nachbeobachtung der UKPD-Studie. Die Vorteile einer frühzeitigen, strikten Blutzuckereinstellung blieben auch während der nachfolgenden Jahre erhalten, obwohl sich die glykämischen Unterschiede zwischen intensiv und konventionell behandelten Patienten nach Studienende wieder angeglichen hatten.

Wegen dieses als "Legacy Effect" oder „Metabolic Memory“ bezeichneten Mechanismus' plädierte Kröger für den früheren Einsatz von Kombinationstherapien. Er sollte erfolgen, sobald erkennbar ist, dass sich durch eine Monotherapie zusätzlich zu Diät und Bewegung keine ausreichende Blutzuckersenkung erreichen lässt. „Mit den verfügbaren oralen Monotherapien kommen wir gegen die Hauptdefekte des Typ-2-Diabetes nicht an", betonte der Experte. Mit einer Kombinationstherapie aus Sitagliptin plus Metformin (z. B. Velmetia ${ }^{\circledR}$ ) sei es dagegen möglich, die Funktionsstörung der Inselzellen, die Insulinresistenz sowie die erhöhte Glukoseproduktion in der Leber gleichzeitig zu behandeln und die Glukosespiegel besser zu kontrollieren als mit den Monotherapien.

\section{Synergistische Effekte} von Metformin und Gliptin

Sitagliptin hemmt die DPP-4, das Abbauenzym der aktiven Inkretine. Dadurch wird in Abhängigkeit von erhöhten Glukosespiegeln die Insulinfreisetzung aus den Betazellen gesteigert und die Glukagonausschüttung aus den Alphazellen des Pankreas vermindert. Metformin unterdrückt vor allem die Glukoseproduktion in der Leber und senkt die periphere Insulinresistenz. „Die gemeinsame Gabe von Sitagliptin und Metformin zeigt eine mehr als additive Wirkung auf die Konzentration des aktiven GLP-1 vor und nach einer Mahlzeit", betonte Kröger.

Dass die Kombination sich auch günstig auf das Körpergewicht und das Risiko für Hypoglykämien auswirkt, bestätigen die Ergebnisse einer 52-wöchigen Studie: Während die Patienten unter Metformin plus Glipizid durchschnittlich 1,1 kg zunahmen, ging das Körpergewicht unter Metformin plus Sitagliptin um 1,5 kg zurück. Mit Glipizid erlitten 32\% der Diabetiker Hypoglykämien, mit Sitagliptin nur 5\%.

\footnotetext{
- Birgit Matejka

Quelle: Fortbildungsveranstaltung: „Diabetes in Balance“, München, 2009 (Veranstalter: Berlin-Chemie)
} 